\title{
Capacity of Visual Classical Conditioning in Drosophila Larvae
}

\author{
Alina M. H. J. von Essen \\ University of Fribourg
}

\author{
Dennis Pauls \\ Philipps-University Marburg
}

\author{
Andreas S. Thum and Simon G. Sprecher \\ University of Fribourg
}

\begin{abstract}
Vision is an ancient sense essential for various aspects of animal behavior. Visual information not only leads to immediate, temporary, and rapid behavioral responses but also has lasting effects. Naïve behavioral responses to light are not always identical but can be altered based on positive or negative experience- a process defined as visual learning. In this study, Drosophila larvae were used as a simple model to study visual classical conditioning. We show that larvae are able to associate positive or negative cues with either light or darkness, thus changing their native light-preference. This effect can be robustly provoked through gustatory stimuli and electric shock. We further show that light can not only be used as a conditioned stimulus but also as an unconditioned stimulus, as punishment in the olfactory classical conditioning procedure, possibly forming two different kinds of memories. Our findings show that even though larvae show a strong naïve response when exposed to light, the animals display a comparably large repertoire of visual memories that can be formed. Therefore, our study provides an impacting entry point into the genetic dissection of the neuronal circuit that underlies different types of visual learning.
\end{abstract}

Keywords: Drosophila, visual learning, classical conditioning, larval behavior

Learning and memory affect fundamental aspects of everyday life. Defects in these brain functions are correlated with serious human neurodegenerative conditions such as Alzheimer's disease (Hirth, 2010; Xia, 2010; Ashe \& Zahs, 2010). Therefore, resolving the anatomical and molecular basis of higher brain functions remains a scientific goal of wide interest. Toward this aim, different animal models have been used to study learning and memory, including the fruit fly, Drosophila melanogaster (Heisenberg, 2003; Keene \& Waddell, 2007; Fiala, 2007). Drosophila shares many organizational features with the mammalian central nervous system. However, the adult Drosophila brain consists of up to 10,000 times less neurons compared with the mammalian brain

Alina M. H. J. von Essen, Andreas S. Thum, and Simon G. Sprecher, Department of Biology, Institute of Cell and Developmental Biology, University of Fribourg, Fribourg, Switzerland; Dennis Pauls, Department of Biology, Institute of Neurobiology/Ethology, Philipps-University Marburg, Marburg, Germany.

Andreas S. Thum is now at the Department of Biology, University of Konstanz, Konstanz, Germany.

We thank our colleagues of the Thum lab, Stocker lab, and Sprecher lab for fruitful discussions and helpful comments on the manuscript. This work was financially supported by the Swiss National Science Foundation (PP00P3_123339 to S. G. S. and 31003A_132812 to A. S. T.).

Correspondence concerning this article should be addressed to Simon G. Sprecher, Department of Biology, Institute of Cell and Developmental Biology, University of Fribourg, Ch. du Musée 10, CH 1700 Fribourg, Switzerland. E-mail: simon.sprecher@unifr.ch
(Nassif, Noveen, \& Hartenstein, 2003). The brain of the Drosophila larva is at least one order of magnitude simpler in complexity in terms of neuron number and connectivity than its adult counterpart. In striking contrast to its simplicity, it displays a wide range of a behavioral repertoire, which makes the Drosophila larva an excellent model system to study different aspects of learning and memory (Gerber \& Stocker, 2007).

Two important functions rely on the larval eye, also termed Bolwig's organ (BO). When exposed to light, Drosophila larvae display striking negative phototaxis (Sawin-McCormack, Sokolowski, \& Campos, 1995). Another capacity of the visual system is the light-dependent entrainment of the molecular clock to control the circadian rhythm, which results in alternating degrees of photonegative behavior over the course of the day (Mazzoni, Desplan, \& Blau, 2005). In contrast to these well-established fields, only little is known in regard to the capacity on larval visual associative learning (Gerber et al., 2004; Knight, Iliadi, Charlton, Atwood, \& Boulianne, 2007).

Learning and memory in Drosophila larvae has been studied mainly for classical olfactory conditioning. Thereby, animals can learn to avoid an odor (the conditioned stimulus [CS]) that was paired with an aversive unconditioned stimulus (US; e.g., high salt concentrations, quinine, or electric shock). Conversely, if the same odor (CS) is paired with an appetitive US (e.g., sugar), animals develop a preference for it (Gerber \& Stocker, 2007; Pauls, Pfitzenmaier, et al., 2010). Thus, depending on previous experience, the same odor can trigger either avoidance or attraction (Gerber \& Hendel, 2006). In contrast, visual learning in Drosophila larvae was only demonstrated when light or darkness was paired with 
positive sugar reinforcement using individual larvae. Using the same protocol, negative reinforcement (salt and quinine) did not lead to aversive visual associative learning (Gerber et al., 2004).

In the present study, we further explore sensory modalities for visual classical conditioning. We show that Drosophila larvae are able to learn visual cues in many more different contexts than previously assumed. We established experimental paradigms using mass assays in which larvae associate light information with gustatory, olfactory, and electric shock stimuli. This allows a direct comparison with recent studies on olfactory associative learning (Yarali, Hendel, \& Gerber, 2006; Kaun, Hendel, Gerber, \& Sokolowski, 2007; Honjo \& Furukubo-Tokunaga, 2009; Pauls, Pfitzenmaier, et al., 2010; Pauls, Selcho, Gendre, Stocker, \& Thum, 2010; Saumweber, Husse, \& Gerber, 2011). As proof of principle, we confirm that light can be used as CS and sugar as rewarding reinforcer, as previously shown in single larva assays by Gerber and coworkers (2004). Next, we demonstrate that high salt concentrations can trigger visual associative learning in an aversive gustatory learning paradigm. We further show that aversive visual associative learning is not constrained to gustatory reinforcement by successfully using electric shock as negative reinforcement. Interestingly, we found that light itself can also be used as punishing US in olfactory learning, changing the preference for one odorant after being paired with light.

Thus, the same stimulus, namely light, can act as conditioned and unconditioned stimulus in the classical conditioning procedure of Drosophila larvae, raising the question of how reward, punishment, and visual information is represented and converged within the neuronal circuit of the larval brain. The possibility to switch between the different learning paradigms, together with the numerical simplicity and functional amenability by genetic intervention of the larval visual circuit, provides an exceptional model to study the fundamental mechanisms of associative visual learning and memory from the cellular up to the behavioral level.

\section{Method}

\section{FLY Strains}

Canton S wild-type strains were kept in mass culture at $25^{\circ} \mathrm{C}$ on standardized cornmeal medium under a 12-hr light-dark cycle. Adults were allowed to lay eggs for $48 \mathrm{hr}$ and were transferred to fresh food vials every second day. Experiments were performed with early-feeding third-instar larvae (96 to $110 \mathrm{hr}$ after egg laying) to avoid that larvae already switched their photobehavior, as described in Sawin-McCormack et al. (1995). Groups of 30 larvae were taken from the food and briefly washed in tap water before the experiment was started.

\section{Experimental Setup}

To perform visual learning experiments, we established the following parameters. We illuminated the agarose plates with white light from above, which avoids larvae crawling up to the lid of the petri dish. We used a light intensity of 760 lux, a value between the threshold to detect light in Drosophila larvae (SawinMcCormack et al., 1995) and a daylight illumination, which might activate other then the Bolwig's organ's photoreceptors (Xiang et al., 2010). The dark preference was more stable on quadrant plates over 5 min compared to half-illuminated agarose plates (data not shown). Therefore, we used quadrant plates for all our learning experiments.

\section{Assay Plates, Light, Odors, and Reinforcer}

All experiments were performed with LED lamps (OSARAM LED, 80012 White), which illuminated the assay plates with white light of 760 lux from the top. The temperature was controlled throughout all experiments between 22 and $23{ }^{\circ} \mathrm{C}$. Petri dishes (85-mm diameter; Greiner Bio-One GmbH, 4550 Kremsmeinster, Austria) were filled with a thin layer of agarose solution (2.5\%; SIGMA Sigma-Aldrich, 9471 Buchs, Switzerland A5093-500G) boiled in a microwave oven. The plates were stored at room temperature and used the same day or on the following day. To keep one half or two quarters of a test plate in the darkness, the lid was covered with black tape. Depending on the learning paradigm, we added fructose (2M, FLUKA Sigma-Aldrich, 9471 Buchs, Switzerland) or sodium chloride (1.5M, FLUKA Sigma-Aldrich, 9471 Buchs, Switzerland) to the agarose solution as a positive or negative gustatory reinforcer, respectively. The olfactory learning paradigm was performed using $10 \mu \mathrm{l}$ of either pure benzaldehyde (BA, FLUKA Sigma-Aldrich, 9471 Buchs, Switzerland) or diluted amylacetate (AM, FLUKA Sigma-Aldrich, 9471 Buchs, Switzerland, 1:250 in paraffin oil, FLUKA Sigma-Aldrich, 9471 Buchs, Switzerland) filled into custom-made Teflon containers of $4.5-\mathrm{mm}$ diameter with perforated lids, as described in Gerber and Stocker (2007). For the learning paradigm using electric shocks, we placed two semicircular copper electrodes of 1-mm diameter and 70-mm length opposite to each other in the petri dish. The electrodes were adjusted to the petri dish such that the distance between them was $5 \mathrm{~cm}$ at their ends and $7.5 \mathrm{~cm}$ in the middle. The electrodes were completely covered with a thin layer of agarose solution (2.5\%), as described in Pauls, Pfitzenmaier, et al. (2010). We used a variable isolating transformer (Müter RTT3, 0-270V AC, 2.5A, 675VA) to apply electric shocks.

\section{Preference Tests and Learning Experiments}

Preference tests. As indicated in Figure 2, preference tests were performed either on test plates where two quarters are dark and two quarters are illuminated, or on test plates where one half was dark and the other half was illuminated. Depending on the experiment, salt or sugar was added to the agarose. Thirty larvae were collected from the food and briefly washed in tap water. Using a wet paintbrush, all 30 larvae were carefully placed in the center of the test plate and were left to move freely. After $5 \mathrm{~min}$, the experimenter quickly counted the number of larvae in the darkness and in the light. A preference index (PREF) for the darkness was calculated as follows:

$$
\operatorname{PREF}_{(\text {darkness) }}=(\text { dark }- \text { light }) / \text { total }
$$

Visual learning with gustatory reinforcement. Two groups of 30 larvae were simultaneously trained under discriminative (in the following, referred to as "reciprocal") conditions (see Figure 1A). Not much is known about the movement of larvae in light and dark conditions; therefore, we cannot say for sure that both conditions are symmetrical. Nevertheless, due to the reciprocal design, the associative nature of the behavioral outcome is assured. One 

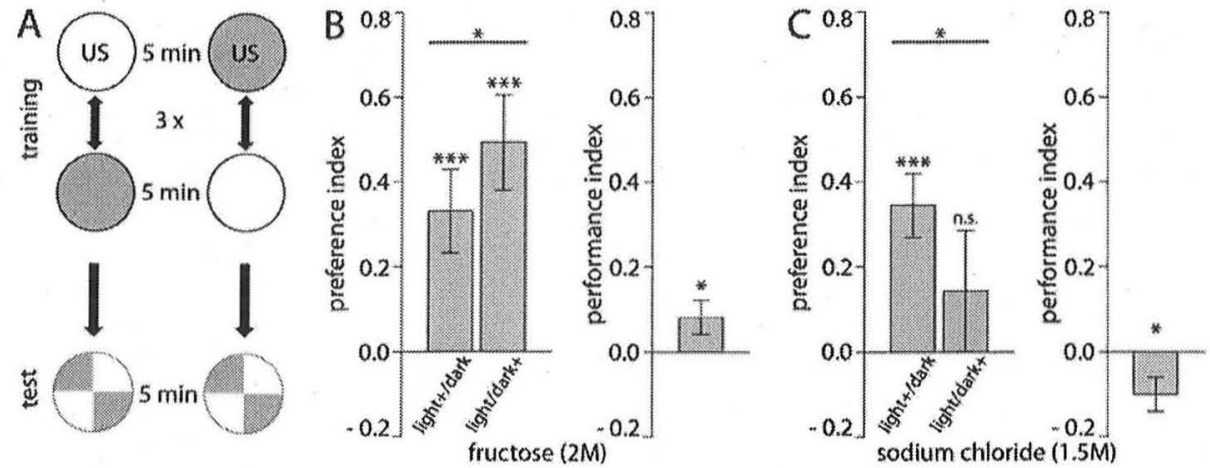

Figure 1. Visual learning with gustatory reinforcement. (A) Reciprocal training protocol pairing light as CS with a gustatory reinforcer (US) and presenting darkness without reinforcer or vice versa. After the training, a light-preference test was performed on neutral or salt-containing quarter plates, respectively. (B) The dark preference after light $+/$ dark training (mean PREF $=0.3296 ; p=.000724)$ is significantly different $(p=$ .02011) from the dark preference of larvae that received light/dark+training (mean PREF $=0.4922$; $p=.0008876$ ). Larvae were able to associate light with fructose as appetitive reinforcer (mean PI $=0.08 ; p=$ $.03015 ; N=15$ ). (C) The dark preference after light $+/$ dark training (mean PREF $=0.3440 ; p=.000724$ ) is significantly different $(p=.02931)$ from the dark preference of larvae that received light/dark + training (mean $\mathrm{PREF}=0.1423 ; p=.1161$ ). Larvae were able to associate darkness with salt as aversive reinforcer (mean PI $=$ $-0.1 ; p=.03315 ; N=15) .{ }^{*} p<.05 .{ }^{* * *} p<.01 .{ }^{* * * *} p<.001$.

group was exposed to light that was rewarded with fructose for 5 $\mathrm{min}$, and then transferred using a wet paintbrush for another $5 \mathrm{~min}$ to a second agarose plate in the darkness, and was not paired with a reinforcer (light $+/$ dark). The second group received training under reciprocal conditions (light/dark + ). After three training cycles, a choice test was conducted on a neutral test plate where two quarters were illuminated and two quarters were dark. The amount of larvae in each quarter was counted after $5 \mathrm{~min}$. From both reciprocally trained groups, we calculated the PREF for the darkness as
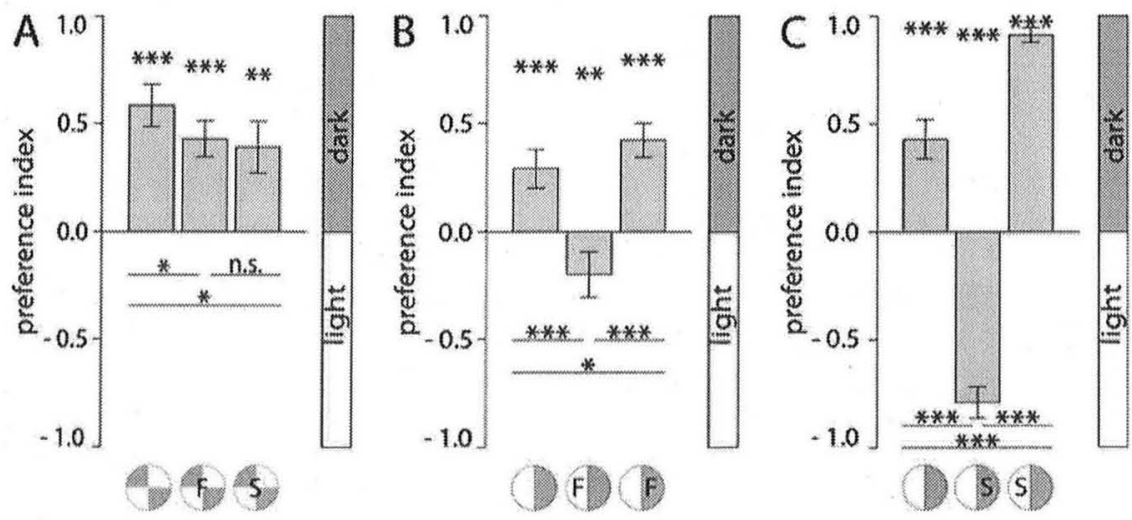

Figure 2. Gustatory input alters the dark preference. (A) Larvae, when tested on quarter test plates containing neutral agarose, show a dark preference (mean PREF $=0.58 ; p=.0007122 ; N=15$ ). Compared to the dark preference on neutral test plates, the dark preference is significantly reduced $(p=.04104)$ on test plates containing fructose (mean PREF $=0.43 ; p=.0007087 ; N=15$ ). The dark preference is significantly reduced $(p=.03713)$ on test plates containing salt (mean PREF $=0.39 ; p=.001088 ; N=15)$ when compared with neutral test plates. (B) A dark preference can be observed for larvae if tested on pure agarose (mean PREF = $0.29 ; p=.0008855 ; N=15$ ), whereas a preference for light can be observed when the fructose-containing half of the agarose is exposed to light (mean PREF $=-0.20 ; p=.004182 ; N=15$ ). The dark preference is enhanced ( $p=.1874$ ) when the fructose-containing side is presented in the darkness (mean PREF $=0.42 ; p=.0007175$; $N=15$ ). (C) A dark preference can be observed for larvae if tested on pure agarose plates (mean PREF $=0.43$; $p=.0006998 ; N=15$ ), whereas a preference for light can be observed when the salt-containing half of the agarose is presented in the darkness (mean PREF $=-0.79 ; p=.000662 ; N=15$ ). The dark preference is enhanced $\left(p=3.113 \times 10^{-06}\right)$ when the salt-containing half of the agarose is presented in the light (mean PREF $=0.91 ; p=.0006928 ; N=15) .{ }^{*} p<.05 .{ }^{* *} p<.01 .{ }^{* * *} p<.001$. 


$$
\operatorname{PREF}_{(\text {darkness })}=(\text { dark }- \text { light }) / \text { total }
$$

and then compiled the performance index (PI) from both groups:

$$
\begin{aligned}
\mathrm{PI}=\left(\left(\mathrm{PREF}_{\text {(darkness) }} \operatorname{light} / \mathrm{dark}+\right)\right. & \\
& \left.-\left(\mathrm{PREF}_{(\text {darkness })} \operatorname{light}+/ \text { dark }\right)\right) / 2
\end{aligned}
$$

Positive values of the PI represent appetitive memories, whereas negative values represent aversive memories like for salt learning. Experiments using salt as negative gustatory reinforcer were performed in the same way as the experiment using fructose as positive gustatory reinforcer described previously. Despite a strong downregulation of feeding, it could be shown that a salt concentration of $1.5 \mathrm{M}$ has an aversive reinforcing effect in the (olfactory) learning process of Drosophila larvae (Hendel et al., 2005).

Visual learning using electric shock as reinforcer. Similar to Pauls, Pfitzenmaier, et al. (2010), a group of 30 larvae was tested for visual-electric shock learning using a nonreciprocal paradigm. The initial dark preference was tested on a quadrant test plate (see Figure $3 \mathrm{~A}$ ). After 5 min, the larvae in the light and dark quarters were counted and the PREF was calculated as described in the Preference Test section. Directly after the pretest, the larvae were transferred to an agarose plate in the darkness for $1 \mathrm{~min}$, and in the last $30 \mathrm{~s}$, were paired with an electric shock. Afterward, larvae were left for $5 \mathrm{~min}$ without application of electric shocks in the light. After repeating the training cycle 10 times, a final choice test with a quadrant test plate was made for $5 \mathrm{~min}$. Larvae in the illuminated and dark quarters were counted and the PREF was calculated. The $\triangle \mathrm{PREF}$ of the nonreciprocal design is calculated as follows:

$$
\triangle \mathrm{PREF}=\mathrm{PREF}_{\text {pre-test }}-\mathrm{PREF}_{\text {test }}
$$

We performed three control experiments to exclude that one of the stimuli presented during the conditioning process (changing light conditions, exposure to electric shocks and handling) influences the light preference in a nonassociative manner. We run the same training procedure as described in this section, while omitting either light exposure, electric shock, or both. Only an unaltered dark preference in the control experiments showed the associative character of the obtained $\triangle \mathrm{PREF}$ where all stimuli were present.

Olfactory learning using light as reinforcer. Based on the assay described by Gerber and Stocker (2007), two groups of 30 larvae were simultaneously trained under two converse conditions (see Figure 4A). One group was exposed to BA, which was punished by light for $5 \mathrm{~min}$, and then transferred to the darkness that was paired with $\mathrm{AM}(\mathrm{BA}+/ \mathrm{AM})$ for another $5 \mathrm{~min}$. The other group was trained reciprocally $(\mathrm{BA} / \mathrm{AM}+)$. After three training cycles, a choice test on which BA and AM were presented on opposite sites on the agarose plate was made in the light. After 5 min, larvae on each side of the test plate were counted and the PREF was calculated

$$
\operatorname{PREF}_{(\mathrm{BA})}=(\mathrm{BA}-\mathrm{AM}) / \text { total }
$$

and the PI was compiled from the reciprocal groups

$$
\mathrm{PI}=\left(\left(\mathrm{PREF}_{(\mathrm{BA})} \mathrm{BA}+/ \mathrm{AM}\right)-\left(\mathrm{PREF}_{(\mathrm{BA})} \mathrm{BA} / \mathrm{AM}+\right)\right) / 2
$$

Again, positive values of the PI represent appetitive memories, whereas negative values represent aversive memories.

\section{Data Analysis}

R language version 2.10.1 and Adobe Photoshop CS3 version 10.0.1 was used for statistical computing and graphical representation. We applied the Wilcoxon signed-rank test to verify a statistically significant difference from zero of one set of data, and the Wilcoxon rank-sum test to compare a statistical significant
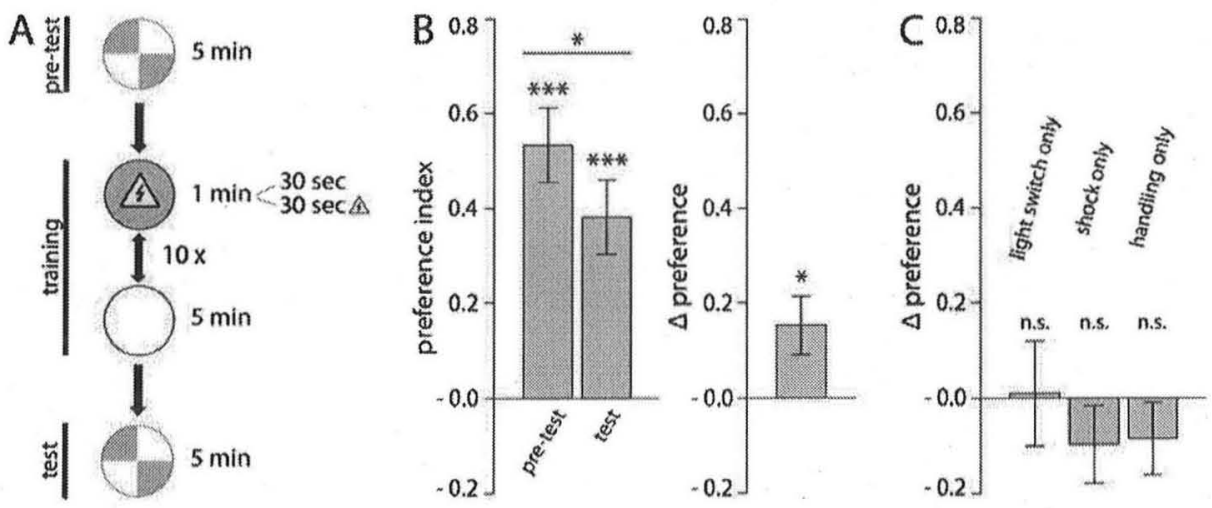

Figure 3. Visual learning with electric shock reinforcement. (A) Nonreciprocal training protocol pairing electric shock with darkness. Prior to the conditioning phase and afterward, a light preference test was completed. The difference of the preferences indicates the performance ( $\triangle \mathrm{PREF}$ ). (B) Using 10 training cycles, the naïve light preference (mean PREF $=0.53 ; p=.0007069, N=15)$ is significantly reduced $(p=.01595$ ) compared to the light preference after training (mean PREF $=0.38 ; p=6.104 \times 10^{-05} ; N=15$ ). The resulting $\triangle \mathrm{PREF}$ is significantly above chance level (mean $\triangle \mathrm{PREF}=0.15 ; p=.03801$ ). (C) The control experiments revealed no change in the light preference before and after the conditioning phase, when the electric shocks ( $p=$ $.977 ; N=12)$, the light switch $(p=.1919 ; N=10)$, or both $(p=.1928 ; N=12)$ were omitted. ${ }^{*} p<.05 .{ }^{* *} p<$ $.01 .{ }^{* * *} p<.001$. 

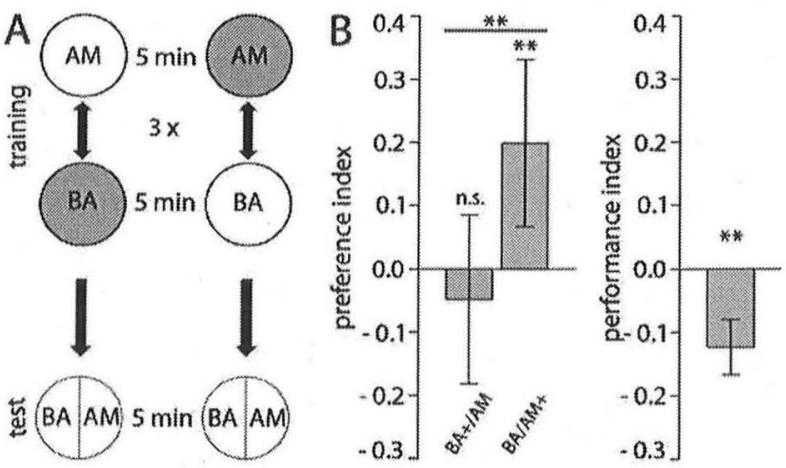

Figure 4. Olfactory learning using light as reinforcer. (A) Reciprocal training protocol pairing either benzaldehyde (BA) or amylacetate (AM), with either light or darkness as US. After the conditioning phase larvae undergo a choice test for the two odorants. (B) The mean preference for BA after receiving $\mathrm{BA}+/ \mathrm{AM}$ training is $-0.04873(p=.2868)$, which is significantly different ( $p=.004695)$ from the BA preference of larvae that received $\mathrm{BA} / \mathrm{AM}+$ training (mean $\mathrm{PREF}=0.1984 ; p=.004221$ ). The punishment of one odorant with light affects the final odor preference which is shown by the mean PI of -0.12 , which is significantly different from zero $(p=.008308 ; N=20) .{ }^{* *} p<.01$.

difference between two groups of data. Significance levels are $p<$ $.05\left(^{*}\right), p<.01\left(^{* *}\right)$, and $p<.001\left(^{* * *}\right)$.

\section{Results}

\section{Light as Conditioned Stimulus}

Visual learning with gustatory reinforcement. Naïve Drosophila larvae show a stereotypic and robust avoidance response when exposed to light; we will use the term "dark preference" to describe this aversive light-dependent behavior. If larvae are able to form associative memories by light exposure (or darkness) with a negative or positive sensory cue, dark preference will be significantly changed after training (Gerber et al., 2004; Knight et al., 2007). First, we asked if the dark preference could be changed by using sugar as rewarding US, similar to Gerber et al. (2004). We modified the paradigm using a mass assay (30 larvae) of three training trials of $5 \mathrm{~min}$ each, as widely used for olfactory associative learning (Gerber \& Stocker, 2007). Second, we asked if the dark preference could be changed by salt using the same mass assay. Two reciprocally trained groups of 30 larvae were exposed to light or darkness paired with either $2 \mathrm{M}$ fructose or $1.5 \mathrm{M}$ sodium chloride as appetitive or aversive gustatory reinforcer, respectively (Figure 1A). The dark preference (PREF) of each reciprocal trained group was calculated, and the difference of the dark preference of the two reciprocally trained groups was quantified as performance index (PI; see Method section). Using fructose as reward in appetitive visual learning, the dark preference of the group receiving light $+/$ dark training is significantly different $(p=$ .02011) from the dark preference of the group that received light/ dark + training. The mean PI of 0.08 is significantly above chance level (Figure 1B; $p=.03015$ ). We next tested, in the aversive gustatory learning paradigm, if salt can be used as US to trigger visual learning when tested on a plate containing salt. Here we obtained a significant difference $(p=.02931)$ in the final dark preference of the reciprocal trained groups. The mean performance index of -0.10 is significantly different from zero (Figure 1C; $p=$ .03315). Thus, larvae are able to associate light with sugar as positive gustatory reward, as well as high salt concentrations as negative gustatory punishment, with moderate learning scores. We revisited light-salt learning, although Gerber et al. (2004) could not find significant aversive visual learning. Recent findings of the same group revealed that memory recall is motivation-dependent: Aversive olfactory salt memory is recalled only in a salt environment but not in a neutral background (Gerber \& Hendel, 2006; Niewalda et al., 2008). Thus, if larvae were tested on a plate containing salt, they are indeed able to show an altered light response.

Gustatory input alters the dark preference. The exposure to several distinct stimuli may affect the response of an animal. Thus, the presence of sugar or salt as gustatory stimuli may alter the preference for darkness. We therefore tested whether our learning experiments provide a pure measure of associative learning or if nonassociative effects contribute to the PI. We performed dark-preference tests on agarose plates containing $2 \mathrm{M}$ fructose or $1.5 \mathrm{M}$ sodium, as used in the learning experiment. Compared to the dark preference on neutral agarose plates, we observed a significantly reduced dark preference when fructose or salt was present during the test $(p=.04104$ and $p=.03713$, respectively; Figure $2 \mathrm{~A})$. Thus, the presence of fructose or salt alters the dark preference.

To further assess the weight of gustatory and light stimuli presented in the learning paradigm, we tested larvae on agar plates where half of the plate contained either fructose or salt. On neutral plates, larvae show a strong dark preference (Figure 2B; PREF = $0.29 ; p=.0008855$ ). Contrary, when the illuminated half of the agarose plate contains fructose, larvae prefer light to dark (Figure $2 \mathrm{~B}$; PREF $=-0.20 ; p=.004182$ ). Thus, if given the choice between fructose or darkness, the animal prefers fructose, indicating that larval behavior is mainly triggered by gustatory information, at least in the used intensities. Conversely, when fructose is presented in darkness (PREF $=0.42 ; p=.0007175)$, the dark preference is significantly enhanced $(p=.01874)$ when compared with the dark preference obtained on neutral agarose plates (Figure 2B). When the whole test plate was illuminated or the whole test plate was in darkness, larvae showed a clear preference for fructose (data not shown). Thus, sugar preference was not changed by the presence or absence of light, while the preference for darkness was changed by the presence of sugar (Figure 2, A and B). Interestingly, the effect is even stronger when salt is present. As shown before, larvae usually avoid light on a pure agarose plate (Figure 2C; PREF $=0.42 ; p=.0006998$ ). However, if darkness is paired with salt, larvae crawl toward the illuminated half of the plate (Figure 2C; PREF $=-0.79 ; p=.000662$ ). The preference for darkness is significantly enhanced $\left(p=3.113 \times 10^{-06}\right)$ when salt is presented in the light exposed half (Figure 2C; PREF $=$ $0.91 ; p=.0006928)$. When the whole test plate was illuminated or the whole test plate was in darkness, no change of the salt avoidance was observed (data not shown). Thus, the presence of a gustatory stimulus indeed alters the dark preference, which may affect the performance in experiments where larvae are tested on salt-containing-agar plates. Due to the reciprocal design of the 
learning experiments described (Figure 1C), the moderate changes in behavior are clearly associative.

Visual learning using electric shock as reinforcer. In adult Drosophila, electric shock is often used as negative reinforcer in olfactory conditioning (McGuire, Deshazer, \& Davis, 2005; Gerber, Stocker, Tanimura, \& Thum, 2009). Similarly in larvae, electric-shock-reinforced olfactory conditioning provides reliable learning scores in olfactory learning (Aceves-Pina and Quinn, 1979; Heisenberg, Borst, Wagner, \& Byers, 1985; Tully, Cambiazo, \& Kruse, 1994; Khurana, Abu Baker, \& Siddiqi, 2009; Pauls, Pfitzenmaier, et al., 2010). Therefore, we adapted an electric shock paradigm for visual learning that allows a comparison with classical olfactory learning (Pauls, Pfitzenmaier, et al., 2010). We applied a nonreciprocal mass assay using 10 training trials of 1 min, as described for olfactory learning. The learning paradigm starts with a dark-preference test prior to the conditioning phase. Subsequently, the first training cycle consist of $1 \mathrm{~min}$ in the darkness, whereas the last $30 \mathrm{~s}$ were paired with an electric shock. After the electric shock, larvae stayed in light to recover for $5 \mathrm{~min}$ from the shock before the training cycle started again. After 10 repetitions, the dark preference was tested. The performance index is calculated by the difference of the dark preference prior and after the conditioning phase. We did not observe significant learning with five training cycles (data not shown), while 10 training cycles led to a robust change in the dark preference. The dark preference after electric shock training (Figure 3B; $P R E F=0.38$; $\left.p=6.104 \times 10^{-05}\right)$ was significantly reduced $(p=.01595)$ when compared with the dark preference of naïve animals before training (Figure 3B; PREF $=0.53 ; p=.0007069$ ). The resulting $\triangle \mathrm{PREF}$, which indicates associative learning, is with 0.15 significantly different from zero (Figure 3B; $p=.03801$ ). Appropriate control experiments demonstrate that learning is purely associative and not due to nonassociative effects: If training consisting of only light exposure $(p=.977)$, electric shock $(p=.1919)$, or handling ( $p=.1928)$, no significant change in the dark preference occurred (Figure 3C). An additional control experiment, in which CS and US are presented in an unpaired manner, provides further support for the associative nature of the behavioral output. With slightly adjusted settings (see Supplementary Figure 1), we repeated the electric shock conditioning procedure, pairing CS and US. The dark preference decreased significantly $(p=.02454)$ after darkness was repeatedly paired with electric shock $(\triangle \mathrm{PREF}=0.1696$; $p=.01025)$. Using the unpaired protocol, in which CS and US are presented one after another, the dark preference does not change ( $p=.693$ ) before and after the training. Thus, no association of CS and US was observed ( $\triangle \mathrm{PREF}=0.00296 ; p=.7332$; see Supplementary Figure 1). Taken together, the results show that larvae are able to form negative associations between visual information and electric shock.

\section{Light as Unconditioned Stimulus}

Olfactory learning using visual reinforcement. We have shown that light can act as a CS when reinforced by gustatory reward or punishment (see Figures 1 and 2) or electric shock punishment (see Figure 3). The naïve behavior of larvae to light is avoidance; therefore, we asked if light could be used as aversive US to condition another stimulus. In the larval olfactory classical conditioning procedure, it has been shown that different negative reinforcers, such as salt, quinine, or electric shock, form aversive associations when paired with an odorant (Gerber \& Stocker, 2007; Pauls, Selcho, et al., 2010). We addressed if light could also be used as aversive US in the olfactory classical conditioning procedure. Accordingly, we adapted a widely used mass assay protocol for olfactory learning (Hendel et al., 2005; Yarali et al., 2006; Selcho, Pauls, Han, Stocker, \& Thum, 2009). We assessed two reciprocally trained groups of larvae, in which one odorant (either benzaldehyde $[\mathrm{BA}]$ or amylacetate $[\mathrm{AM}]$ ) was paired with light, and the second odorant was presented in darkness, and vice versa. After three training cycles, each group of reciprocally trained larvae was given the choice between both odorants (Figure $4 \mathrm{~A})$. The group of larvae that received $\mathrm{BA}+/ \mathrm{AM}$ training had a significantly lower $(p=.004695)$ preference for BA (PREF $=$ $-0.0487)$ than larvae that received $\mathrm{BA} / \mathrm{AM}+$ training $(\mathrm{PREF}=$ 0.1984; Figure 4B). As for the reciprocal light learning assays, the final difference in the odor-preference of the two reciprocally trained groups is quantified by the PI. Larvae avoid the odorant that was during training paired with light (Figure 4B; PI $=-0.12 ; p=$ .008308). Thus, light can also be used as an aversive US for olfactory associative learning. In order to test if larvae avoid the light-associated odorant or are attracted to the dark-associated odorant, we performed the same learning experiment, but during the test situation, only the light-associated or the dark-associated odorant was presented. Larvae preferred the dark-associated odor when tested against no odor (mean $\mathrm{PI}=0.14921 ; p=.01245$ ). No significant behavior change was detectable for the odorant paired with light (mean PI $=0.0989 ; p=$ .07879). This experiment suggests that larvae associate an odorant only with the rewarding darkness (see Supplementary Figure 2).

\section{Discussion}

\section{A Comprehensive Set of Paradigms to Examine Light-Related Behavioral Changes in Drosophila Larvae}

We have developed behavioral paradigms to assess visual classical conditioning with several sensory modalities. We have shown that Drosophila larvae can associate visual cues as CS with either appetitive or aversive gustatory reinforcement (see Figure 1). Electric shock learning can lead to lasting alterations in the larva's light avoidance when the shock is repeatedly presented in darkness (see Figure 3). Interestingly, we further found that light also acts as a US, changing the odor-preference of Drosophila larvae (see Figure 4). Taken together, the work presents a comprehensive set of behavior paradigms that allow a detailed analysis on how light leads to lasting changes in larval behavior.

\section{Gustatory Input Interferes With the Dark Preference}

In contrast to associative learning, behavioral responses for a specific sensory cue, such as light, may be changed only by the presence of other sensory stimuli. The main task of the animal during larval stages is constant food uptake and growth of body mass throughout day and night (Ashburner, 1989). Therefore, it is tempting to speculate that gustatory and olfactory stimuli-foodrelated cues-may suppress visual information. This might indeed be the case, as the preference for darkness is significantly reduced when larvae are tested on agarose plates containing fructose (see Figure 2). The same is true when visual information is evaluated in 
the present of salt (see Figure 2). Our data differ from the Gerber et al. (2004) results, possibly due to differences in the experimental setup, such as different light sources, position of the light source, or breeding larvae on different food media. Our findings suggests that gustatory information per se does not inhibit the naïve visual response but, rather, that more complex evaluation of the different sensory modalities triggers the final behavioral output. A similar effect was described recently, where a reduction of the dark preference could be observed when an odorant was presented during a dark preference test (Yarali et al., 2006; Bellmann et al., 2010). Thus, it seems likely that larval attention is selective and less directed at light when gustatory or olfactory stimuli are presented simultaneously with visual stimuli.

\section{Visual Versus Olfactory Classical Conditioning}

The preference of Drosophila larvae for one odorant can be changed according to prior experience. Reward or punishment of one odorant with gustatory cues or electric shock triggers either avoidance or attraction (Gerber \& Hendel, 2006; Pauls, Pfitzenmaier, et al., 2010). We find that dark preference is similarly plastic. The naïve response to light can be changed via appetitive and aversive gustatory stimuli or electric shock (Gerber et al., 2004; Knight et al., 2007; Figures 1 and 3), comparable to olfactory learning. In particular, the similarity of protocols for olfactory learning and visual learning, as demonstrated here, is striking: number of training cycles, number of trained and tested larvae, training time, salt and sugar concentrations, electric shock application, intertrial intervals, and assay assembly. Therefore, even if different sensory modalities are involved in these kinds of assays, the mechanism of forming the association might be similar. This might be especially true for the mechanisms signaling reward and punishment (Schroll et al., 2006; Selcho et al., 2009; Honjo \& Furukubo-Tokunaga, 2009). It seems likely that, similar to aversive olfactory learning, in aversive visual learning, salt also has to be present during the test to trigger the recall of a memory (see Figure 1; Gerber \& Hendel, 2006). This raises the question of whether the same larval brain centers are involved in the formation and recall of visual and olfactory memories. It will be of broad impact to further deepen the analysis in order to compare neuronal substrates having the potential to form a memory trace in the larva.

\section{Visual Input and Neuronal Substrates for Visual Memory Formation}

The larval eye presumably drives the naïve light response, at least at moderate light intensities (Hassan, Iyengar, Scantlebury, Moncalvo, \& Campos, 2005; Keene \& Waddell, 2007; Keene et al., 2011). The larval eye is composed of 12 photoreceptor neurons (PRs); four of them express the blue-sensitive Rhodopsin6 and eight cells express the green-sensitive Rhodopsin5 (Sprecher, Reichert, \& Hartenstein, 2007; Sprecher \& Desplan, 2008). Both PR types project their axons to the larval optic neuropile (LON), the first visual integration center in the brain. In the LON, the PRs contact target neurons, which transfer the light information into higher, yet unknown, brain regions (Sprecher, Cardona, and Hartenstein, 2011). The adult visual memories, like elevation in the panorama and contour orientation, are located within a neuropile called the central complex (CC; Liu et al., 2006). The CC develops and differentiates only during metamorphosis (Varnam, Strauss, Belle, \& Sokolowski, 1996; Renn et al., 1999; YounossiHartenstein, Nguyen, Shy, \& Hartenstein, 2006) and can therefore not establish larval visual memories. What else are the neuronal substrates for visual learning in larvae? First, it is possible that there is a larval specific counterpart of the CC that is yet not described. Second, for larval olfactory learning, a memory trace can be located within a mirror-symmetrical structure of the larval brain, the so-called mushroom bodies (Pauls, Selcho, et al., 2010). Based on the potential of these neurons to form experiencedependent synaptic changes reinforced by gustatory stimuli and electric shock (Heisenberg et al., 1985; Pauls, Selcho, et al., 2010), it is possible that the mushroom body may also act as memory center for different forms of visual learning.

\section{Visual Input: Differentiation Between CS and US}

Formation of visual memory in the larva can be of two types. First, animals learn that a positive or negative cue was paired with light and will therefore increase or decrease their natural preference for darkness (light as CS). Second, since light is, by itself, an aversive stimulus, light can be paired as aversive US with an olfactory cue, which subsequently will be less attractive. This raises the intriguing issue of how the animal is able to use light information at the same time as CS and US, and, in particular, what circuits or neurons are involved in this process. Until recently, the eye was the only identified source of light-derived neuronal information, mediated by photosensory neurons. However, recently, class IV multidendritc neurons on the larval body wall have been shown to be able to elicit light-avoidance responses in response to high light intensities (Xiang et al., 2010). Complementing experiments using optogenetic activation and targeted silencing demonstrated that these class IV multidendritc sensory neurons are also used by larvae to detect nociceptive stimuli (Hwang et al., 2007). Therefore, it is tempting to speculate that class IV multidendritic neurons primarily perceive different types of noxious stimuli and, therefore, that these neurons may encode light as aversive US. It will be of interest to further investigate if the two light-sensing systems, the larval eye and the class IV multidendritic neurons, serve distinct functions for visual learning and if they use overlapping or distinct neuronal pathways.

Taken together, we describe, for the first time, a comprehensive set of behavioral experiments that allow a detailed analysis of visual learning in Drosophila larvae. The simplicity of the larval visual system, combined with the experimental accessibility of Drosophila on the genetic, electrophysiological, and cellular level, make this system suitable for an integrated understanding of visual learning.

\section{References}

Aceves-Pina, E. O., \& Quinn, W. G. (1979, October 5). Learning in normal and mutant Drosophila larvae. Science, 206, 93-96. doi:10.1126/ science.206.4414.93

Ashburner, M. 1989. Drosophila. A laboratory handbook. Cold Spring Harbor, NY: Cold Spring Harbor Laboratory Press.

Ashe, K. H., \& Zahs, K. R. (2010). Probing the biology of Alzheimer's disease in mice. Neuron, 66, 631-645. doi:10.1016/j.neuron.2010.04.031

Bellmann, D., Richardt, A., Freyberger, R., Nuwal, N., Schwarzel, M. Fiala, A., \& Stortkuhl, K. F. (2010). Optogenetically induced olfactory 
stimulation in drosophila larvae reveals the neuronal basis of odoraversion behavior. Frontiers in Behavioral Neuroscience, 4, 27. doi: 10.3389/fnbeh. 2010.00027

Fiala, A. (2007). Olfaction and olfactory learning in Drosophila: Recent progress. Current Opinion in Neurobiology, 17, 720-726. doi:10.1016/ j.conb.2007.11.009

Gerber, B., \& Hendel, T. (2006). Outcome expectations drive learned behaviour in larval Drosophila. Proceedings. Biological Sciences/The Royal Society, 273, 2965-2968. doi:10.1098/rspb.2006.3673

Gerber, B., Scherer, S., Neuser, K., Michels, B., Hendel, T., Stocker, R. F., \& Heisenberg, M. (2004). Visual learning in individually assayed Drosophila larvae. Journal of Experimental Biology, 207, 179-188. doi: $10.1242 /$ jeb.00718

Gerber, B., \& Stocker, R. F. (2007). The Drosophila larva as a model for studying chemosensation and chemosensory learning: A review. Chemical Senses, 32, 65-89. doi:10.1093/chemse/bj1030

Gerber, B., Stocker, R. F., Tanimura, T., \& Thum, A. S. (2009). Smelling, tasting, learning: Drosophila as a study case. Results and Problems in Cell Differentiation, 47, 139-185. doi:10.1007/400_2008_9

Hassan, J., Iyengar, B., Scantlebury, N., Moncalvo, V. R., \& Campos, R. (2005). Photic input pathways that mediate the Drosophila larval response to light and circadian rhythmicity are developmentally related but functionally distinct. Journal of Comparative Neurology, 481, 266-275. doi: $10.1002 /$ cne. 20383

Heisenberg, M. (2003). Mushroom body memoir: From maps to models. Nature Reviews Neuroscience, 4, 266-275. doi:10.1038/nrn1074

Heisenberg, M., Borst, A., Wagner, S., \& Byers, D. (1985). Drosophila mushroom body mutants are deficient in olfactory learning. Journal of Neurogenetics, 2, 1-30. doi:10.3109/01677068509100140

Hendel, T., Michels, B., Neuser, K., Schipanski, A., Kaun, K., Sokolowski, M. B., ... Gerber, B. (2005). The carrot, not the stick: Appetitive rather than aversive gustatory stimuli support associative olfactory learning in individually assayed Drosophila larvae. Journal of Comparative Physiology. A, Sensory, Neural, and Behavioral Physiology, 191, 265-279. doi:10.1007/s00359-004-0574-8

Hirth, F. (2010). Drosophila melanogaster in the study of human neurodegeneration. CNS \& Neurological Disorders Drug Targets, 9, 504523.

Honjo, K., \& Furukubo-Tokunaga, K. (2009). Distinctive neuronal networks and biochemical pathways for appetitive and aversive memory in Drosophila larvae. Journal of Neuroscience, 29, 852-862. doi:10.1523/ JNEUROSCI.1315-08.2009

Hwang, R. Y., Zhong, L., Xu, Y., Johnson, T., Zhang, F., Deisseroth, K., \& Tracey, W. D. (2007). Nociceptive neurons protect Drosophila larvae from parasitoid wasps. Current Biology, 17, 2105-2116. doi:10.1016/ j.cub.2007.11.029

Kaun, K. R., Hendel, T., Gerber, B., \& Sokolowski, M. B. (2007). Natural variation in Drosophila larval reward learning and memory due to a cGMP-dependent protein kinase. Learning \& Memory, 14, 342-349. doi: $10.1101 / 1 \mathrm{~m} .505807$

Keene, A. C., Mazzoni, E. O., Zhen J., Younger, M. A., Yamaguchi, S., Blau J., ... Sprecher, S. (2011). Distinct visual pathways mediate Drosophila larval light avoidance and circadian clock entrainment. Journal of Neuroscience, 31, 6527-6534.

Keene, A. C., \& Waddell, S. (2007). Drosophila olfactory memory: Single genes to complex neural circuits. Nature Reviews Neuroscience, 8, 341-354. doi:10.1038/nrn2098

Khurana, S., Abu Baker, M. B., \& Siddiqi, O. (2009). Odour avoidance learning in the larva of Drosophila melanogaster. Journal of Biosciences, 34, 621-631. doi:10.1007/s12038-009-0080-9

Knight, D., Iliadi, K., Charlton, M. P., Atwood, H. L., \& Boulianne, G. L. (2007). Presynaptic plasticity and associative learning are impaired in a Drosophila presenilin null mutant. Developmental Neurobiology, 67, 1598-1613. doi:10.1002/dneu.20532
Liu, G., Seiler, H., Wen, A., Zars, T., Ito, K., Wolf, R., . . Liu, L. (2006, February 2). Distinct memory traces for two visual features in the Drosophila brain. Nature, 439, 551-556. doi:10.1038/nature04381

Mazzoni, E. O., Desplan, C., \& Blau, J. (2005). Circadian pacemaker neurons transmit and modulate visual information to control a rapid behavioral response. Neuron, 45, 293-300. doi:10.1016/j.neuron .2004 .12 .038

McGuire, S. E., Deshazer, M., \& Davis, R. L. (2005). Thirty years of olfactory learning and memory research in Drosophila melanogaster. Progress in Neurobiology, 76, 328-347. doi:10.1016/j.pneurobio .2005 .09 .003

Nassif, C., Noveen, A., \& Hartenstein, V. (2003). Early development of the Drosophila brain: III. The pattern of neuropile founder tracts during the larval period. Journal of Comparative Neurology, 455, 417-434. doi: $10.1002 / \mathrm{cne} .10482$

Niewalda, T., Singhal, N., Fiala, A., Saumweber, T., Wegener, S., \& Gerber, B. (2008). Salt processing in larval Drosophila: Choice, feeding, and learning shift from appetitive to aversive in a concentrationdependent way. Chemical Senses, 33, 685-692. doi:10.1093/chemse/ bjn037

Pauls, D., Pfitzenmaier, J. E., Krebs-Wheaton, R., Selcho, M., Stocker, R. F., \& Thum, A. S. (2010). Electric shock-induced associative olfactory learning in Drosophila larvae. Chemical Senses, 35, 335-346. doi: $10.1093 /$ chemse/bjq023

Pauls, D., Selcho, M., Gendre, N., Stocker, R. F., \& Thum, A. S. (2010). Drosophila larvae establish appetitive olfactory memories via mushroom body neurons of embryonic origin. Journal of Neuroscience, 30, 10655 10666. doi:10.1523/JNEUROSCI.1281-10.2010

Renn, S. C., Armstrong, J. D., Yang, M., Wang, Z., An, X., Kaiser, K., \& Taghert, P. H. (1999). Genetic analysis of the Drosophila ellipsoid body neuropil: Organization and development of the central complex. Journal of Neurobiology, 41, 189-207. doi:10.1002/(SICI)1097-4695(19991105)41:2 $<189::$ AID-NEU3>3.0.CO;2-Q

Saumweber, T., Husse, J., \& Gerber, B. (2011). Innate attractiveness and associative learnability of odors can be dissociated in larval Drosophila. Chemical Senses, 36, 223-235. doi:10.1093/chemse/bjq128

Sawin-McCormack, E. P., Sokolowski, M. B., \& Campos, A. R. (1995). Characterization and genetic analysis of Drosophila melanogaster photobehavior during larval development. Journal of Neurogenetics, 10 119-135. doi: $10.3109 / 01677069509083459$

Schroll, C., Riemensperger, T., Bucher, D., Ehmer, J., Voller, T., Erbguth, K., . . . Fiala, A. (2006). Light-induced activation of distinct modulatory neurons triggers appetitive or aversive learning in Drosophila larvae. Current Biology, 16, 1741-1747. doi:10.1016/j.cub.2006.07.023

Selcho, M., Pauls, D., Han, K. A., Stocker, R. F., \& Thum, A. S. (2009). The role of dopamine in Drosophila larval classical olfactory conditioning. PLoS One, 4, e5897. doi:10.1371/journal.pone.0005897

Sprecher, S. G., \& Desplan, C. (2008). Switch of rhodopsin expression in terminally differentiated Drosophila sensory neurons. Nature, 454, 533537. doi: $10.1038 /$ nature 07062

Sprecher, S. G., Reichert, H., \& Hartenstein, V. (2007). Gene expression patterns in primary neuronal clusters of the Drosophila embryonic brain Gene Expression Patterns, 7, 584-595. doi:10.1016/j.modgep.2007.01.004

Sprecher, S. G., Cardona, A., \& Hartenstein, V. (2011). The Drosophila larval visual system: High-resolution analysis of a simple visual neuropil. Developmental Biology, 358, 33-43. doi:10.1016/j.ydbio 2011.07.006

Tully, T., Cambiazo, V., \& Kruse, L. (1994). Memory through metamorphosis in normal and mutant Drosophila. Journal of Neuroscience, 14, $68-74$.

Varnam, C. J., Strauss, R., Belle, J. S., \& Sokolowski, M. B. (1996). Larva behavior of Drosophila central complex mutants: Interactions between no bridge, foraging, and Chaser. Journal of Neurogenetics, 11, 99-115. doi: $10.3109 / 01677069609107065$ 
Xia, W. (2010). Exploring Alzheimer's disease in zebrafish. Journal of Alzheimer's Disease, 20, 981-990.

Xiang, Y., Yuan, Q., Vogt, N., Looger, L. L., Jan, L. Y., \& Jan, Y. N. (2010, December 16). Light-avoidance-mediating photoreceptors tile the Drosophila larval body wall. Nature, 468, 921-926. doi:10.1038/ nature09576

Yarali, A., Hendel, T., \& Gerber, B. (2006). Olfactory learning and behaviour are 'insulated' against visual processing in larval Drosophila. Journal of Comparative Physiology. A, Sensory, Neural, and Behavioral Physiology, 192, 1133-1145. doi:10.1007/s00359-006-0140-7
Younossi-Hartenstein, A., Nguyen, B., Shy, D., \& Hartenstein, V. (2006) Embryonic origin of the Drosophila brain neuropile. Journal of Comparative Neurology, 497, 981-998. doi:10.1002/cne.20884 